Article

\title{
Angle-Sensitive Detector Based on Silicon-On-Insulator Photodiode Stacked with Surface Plasmon Antenna
}

\author{
Anitharaj Nagarajan ${ }^{1,2}\left(\right.$, Shusuke Hara ${ }^{3}$, Hiroaki Satoh ${ }^{3,4}\left(\right.$, Aruna Priya Panchanathan ${ }^{2}(\mathbb{D}$ \\ and Hiroshi Inokawa $1,3,4, *$ (D) \\ 1 Graduate School of Science and Technology, Shizuoka University, Hamamatsu 432-8011, Japan; \\ anithr.91@gmail.com \\ 2 Department of Electronics and Communication Engineering, SRM Institute of Science and Technology, \\ Chennai 603 203, India; arunaprp@srmist.edu.in \\ 3 Graduate School of Integrated Science and Technology, Shizuoka University, Hamamatsu 432-8561, Japan; \\ hara.shusuke.14@shizuoka.ac.jp (S.H.); satoh.hiroaki@shizuoka.ac.jp (H.S.) \\ 4 Research Institute of Electronics, Shizuoka University, Hamamatsu 432-8011, Japan \\ * Correspondence: inokawa.hiroshi@shizuoka.ac.jp
}

Received: 5 August 2020; Accepted: 23 September 2020; Published: 28 September 2020

\begin{abstract}
We present a pixel-level angle sensitive detector composed of silicon-on-insulator (SOI) photodiode (PD) stacked with a gold surface plasmon (SP) antenna to affect the direction of the incoming light. The surface plasmons are excited in the grating-type SP antenna and enhance the diffraction efficiency of the grating. The diffracted light is coupled strongly with the propagation light in the SOI waveguide when the phase matching condition is satisfied. The phase matching takes place at a specific angle of light incidence, and the discrimination of the light based on the incident angle is achieved. As spatial patterns in the polar coordinate of the elevation-azimuth angles $(\theta, \phi)$ of the incident light, we present the phase matching condition theoretically, the absorption efficiency in the SOI by simulation, and also the quantum efficiency of the SOI PD experimentally for different SP antennas of one-dimensional (1D) line-and-space (L/S) and two-dimensional (2D) hole array gratings under various polarization angles. $1 \mathrm{D}$ grating offers a polarization sensitive angle detection and 2D grating exhibits angle detection in two orthogonal directions, enabling a polarization independent angle sensitivity. A good agreement among the theory, simulation, and experiment are attained. The proposed device features relatively high quantum efficiency as an angle-sensitive pixel (ASP) and gives wider opportunities in applications such as three-dimensional (3D) imaging, depth-of-field extension, and lensless imaging.
\end{abstract}

Keywords: angle-sensitive pixel; phase matching condition; SOI PD; SP antenna; lensless imaging

\section{Introduction}

The current trend in the research field of advanced image sensors is to focus on the development of pixel-level detectors for computational imaging [1-4]. A traditional camera forms an image by recording only a two-dimensional (2D) intensity map of a scene. A light field camera or plenoptic camera records light field information, which provides a more complete description of a scene than a traditional camera. The light field is the collection of light rays flowing in every direction through every point in space. The light field is defined by the mathematical function called plenoptic function denoted by five-dimensional (5D) intensity data $\mathrm{I}(x, y, z, \theta, \phi)$ [5]. The 5D plenoptic function is the combination of the three spatial coordinates $(x, y, z)$ and the two angle information $(\theta, \phi)$ which represent elevation and azimuth angles, respectively, of the light ray. For simplicity, this function is reduced to 4D data 
$\mathrm{I}(x, y, \theta, \phi)$, by omitting $z$, as the intensity along a ray is a constant [6,7]. Several techniques have been developed to capture the local angular distribution from the light field for many interesting applications. For example, capturing the angle information allows computationally refocusing of the image during post-processing [8]. Furthermore, it also allows 3D imaging, lensless imaging, and so on [9-15]. Recently, a novel CMOS (complementary metal-oxide-semiconductor) image sensor has been developed to detect the angular information as a representative angular sensitive pixel (ASP). This sensor utilizes the Talbot effect between two diffraction gratings stacked over a conventional photodiode and it became possible to measure one angular information $I(\theta)$ of the incident light [16]. However, in order to complete a plenoptic function, it is indispensable to be able to sense two angle information, which includes elevation angle $\theta$ and azimuth angle $\phi$. It was reported that this Talbot pixel has a problem of reduction in quantum efficiency $(\mathrm{QE})$ of $88 \%$ compared to the bare photodiode and was improved to a $55 \%$ reduction rate after the post-fabrication process of ASP; however, it exhibits an angular resolution of $\sim 8^{\circ}$. Moreover, the output response of Talbot pixel is sinusoidal and fades as the angle increases. It requires four pixels to decode single angle information. Hence, the development of CMOS compatible, easily fabricated, high QE possessing features with a less angular resolution and a capability of using a smaller number of pixels to decode single angle information is highly anticipated.

In this article, the performance of incident angle dependence of light sensitivity in our proposed silicon-on-insulator photodiode (SOI PD) with surface plasmon (SP) antenna [17-20] is investigated for one candidate of advanced ASPs. In addition, the incident angle dependence for different incident polarization is also clarified. Our proposed SOI PD with SP antenna basically utilizes the strong coupling between the diffracted light from the grating structure of the SP antenna and the waveguiding modes in the SOI layer. We have investigated the elevation-azimuth angle dependence of the light sensitivity in SOI PD with a SP antenna, but the polarization angle directly depends on the azimuth angle because of our previous measurement configuration with one axis goniometer stage [21,22]. Since such a situation is unnatural, a two-axis goniometer stage is introduced instead of a one axis goniometer stage to evaluate a full elevation-azimuth angle dependence for a specific polarization. In this case, the polarization angle is independent of the azimuth angle, and thus the investigation in this article is close to the actual situation for light detection.

We investigate the directivity of light sensitivity in SOI PD with two types of SP antenna composed of one-dimensional (1D) line-and-space (L/S) grating and 2D hole array grating by using theoretical prediction, electromagnetic simulation, and experimental demonstration. The structure of 1D grating is formed by the periodic arrangement of the Au line and space in one direction. The 2D grating is formed by the superimposition of the 1D grating stacked orthogonally to each other, thereby resulting in a periodic arrangement of holes in both of the directions. A theoretical prediction includes the formalization for representing the peak position for an elevation-azimuth angular distribution of incident light. The physical concept behind this theory is that a higher light sensitivity is achieved at a specific incident angle when the phase matching condition between the diffracted light from the antenna and the propagation light wave in the SOI waveguide is satisfied. The antenna primarily works as a grating coupler and exhibits the angle sensitive characteristics for orthogonal polarized light for the 1D LS grating. In order to realize angle sensitive characteristics with polarization independent behavior, 2D hole array grating is employed, which has periodicity in both of the directions. An electromagnetic simulation based on the finite-difference time-domain (FDTD) method can design and estimate the performances of SOI PD with SP antenna for not only peak positions of the incident angle but also light sensitivity. The monochromatic incident light is tilted and rotated along with the elevation and azimuth angles, $\theta$ and $\phi$, respectively for calculating the light absorption efficiency of the device. The SP antenna over the light sensitive area of the SOI PD is fabricated by using an electron beam lithography technique. The experimental demonstration of the fabricated devices is done by measuring the directivity of quantum efficiency for different polarizations. 


\section{Device Structure and Fabrication Process}

The device structure of the SOI PD with SP antenna is shown in Figure 1a. The lateral p-n junction SOI PD is formed by using commercial SOI wafer. In this work, the typical SOI thickness of $t_{\mathrm{SOI}}=100 \mathrm{~nm}$ was used, since an SOI thickness of below $200 \mathrm{~nm}$ is widely used in SOI integrated circuits. The silicon dioxide $\left(\mathrm{SiO}_{2}\right)$ layer on $\mathrm{SOI} \mathrm{PD}$, which works as a gate oxide, has the thickness of $t_{\mathrm{GOX}}=100 \mathrm{~nm}$. The primary role of the SP antenna is to act as a grating coupler between the incoming light and the lateral propagating light in the SOI layer. The $t_{\mathrm{GOX}}$ was optimized to achieve the maximum QE for an incident wavelength of $700 \mathrm{~nm}$ [17]. The QE is the ratio of the number of photogenerated carrier to the one of incident photons to the device. Without SP antenna, the SOI PD shows low $\mathrm{QE}$ due to the thin $t_{\mathrm{SOI}}$ for light absorption, but the QE can be enhanced by up to $25 \%$ by introducing the SP antenna with $1 \mathrm{D} \mathrm{L} / \mathrm{S}$ gold $(\mathrm{Au})$ grating, as we previously reported [18]. This time, we introduced two types of SP antennas with $\mathrm{L} / \mathrm{S}$ and hole array gratings, and clarified the difference between their characteristics. The L/S grating has a 1D periodic arrangement with a period $p$ and a line width $w$, and the hole array grating has a 2D periodic arrangement of square holes in a square lattice with a period $p$ and a line width $w$, as shown in Figure $1 \mathrm{~b}, \mathrm{c}$, respectively. Au was chosen as the material for a SP antenna because Au has higher durability towards the surface oxidation than that of silver or aluminum. In order to obtain the sufficient adhesion strength between $\mathrm{Au}$ and $\mathrm{SiO}_{2}$, thin titanium (Ti) layer was inserted. The thicknesses of the $\mathrm{Au}$ and Ti used were $t_{\mathrm{Au}}=50 \mathrm{~nm}$ and $t_{\mathrm{Ti}}=5 \mathrm{~nm}$, respectively. The SP antenna acts not only as a grating coupler between incident and laterally propagating waves in the SOI, but also as a gate electrode. When the device is irradiated, the diffracted light from the SP antenna excites the SOI waveguide mode. A large QE can be obtained only if the phase matching condition is satisfied, as will be discussed later. It is also important to apply appropriate bias voltages to the gate and the substrate so that the photocurrent can be maximized by expanding the depletion region to cover the entire light sensitive $\left(\mathrm{p}^{-}\right)$area [18]. The angle dependence in light sensitivity was investigated in this work. The definitions of elevation angle $\theta$, and azimuth angle $\phi$ are shown in Figure $1 \mathrm{~d}$. In addition, the polarization angle $\phi_{\text {pol }}$ with respect to the grating direction is defined in this figure. The incident light is called TE-polarized and TM-polarized when the electric field component coincides with the $x$-direction $\left(\phi_{\text {pol }}=0\right)$ and y-direction $\left(\phi_{\text {pol }}=90^{\circ}\right)$, respectively. The projected elevation angles on $x-z$ and $y-z$ planes, which are $\theta_{x z}$ and $\theta_{y z}$, respectively, are the variables used in the explanation of the physical origin of the peak angle in Section 3 , and are expressed as follows:

$$
\begin{aligned}
& \theta_{x z}=\tan ^{-1}(\tan \theta \cos \phi), \\
& \theta_{y z}=\tan ^{-1}(\tan \theta \sin \phi) .
\end{aligned}
$$

In this paper, the case is discussed for the first time where the polarization angle $\phi_{\text {pol }}$ is independent of $\phi$, whereas $\phi_{\text {pol }}$ was rotated together with $\phi$ in our previous work [21,22].

This SOI PD was fabricated by using the following steps. The first step was to adjust the thickness of the p-type SOI layer through thermal oxidation and removal of the oxide layer. The second step was to pattern the SOI layer by photolithography for the isolation of PDs. The third step was to implant the $\mathrm{BF}_{2}{ }^{+}$and the $\mathrm{As}^{+}$ions to form anode and cathode regions, respectively. The fourth step was to form a gate oxide on the SOI layer by using oxidation and chemical vapor deposition. The final step was to pattern the Au SP antenna by using electron-beam lithography and a lift-off process. The top view of the fabricated device with Au grating stacked over the light sensitive $\mathrm{p}^{-}$area of $50 \times 50 \mu^{2}$ is shown in Figure 2. Since the SP antenna is surrounded by the frame, an electrical contact to the gate electrode is established. This fabricated device is compatible with the CMOS integrated circuit technology except for the usage of $\mathrm{Au}$, and offers good manufacturability as a monolithic device with multiple PDs in a single chip. 


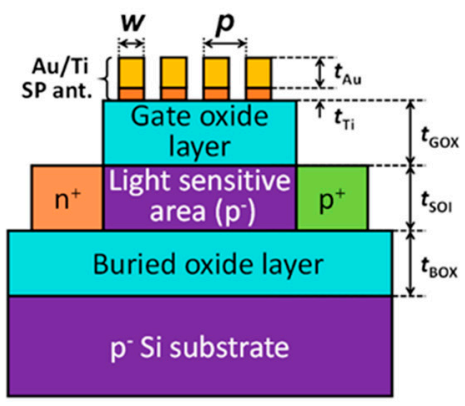

(a)

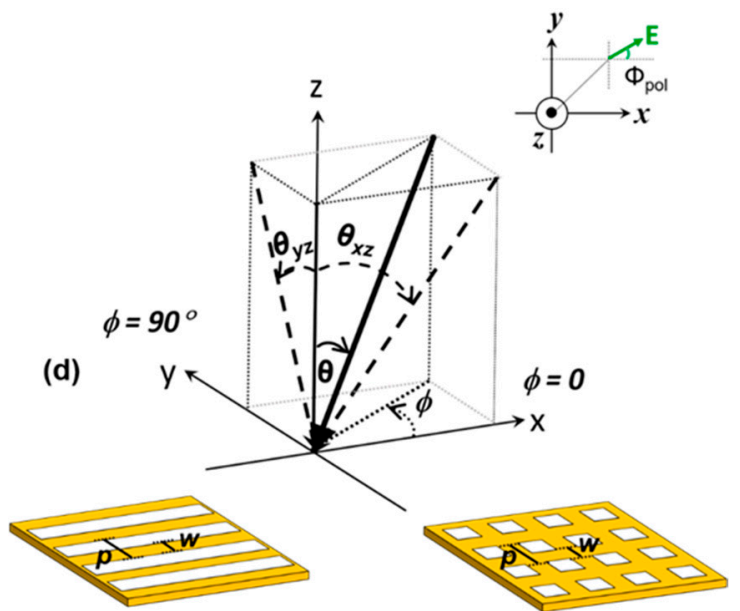

(c)

Figure 1. (a) Cross-sectional view of the silicon-on-insulator (SOI) photodiode (PD) with a surface plasmon (SP) antenna; (b) 1D line and space grating; (c) 2D hole array grating; (d) definitions of azimuth $(\phi)$, elevation $(\theta)$, and polarization $\left(\phi_{\text {pol }}\right)$ angles of the incident light reproduced from [22]. The projected elevation angles $\theta_{x z}$ and $\theta_{y z}$ to $x-z$ and $y-z$ planes, respectively, are also clarified for theoretical discussions.

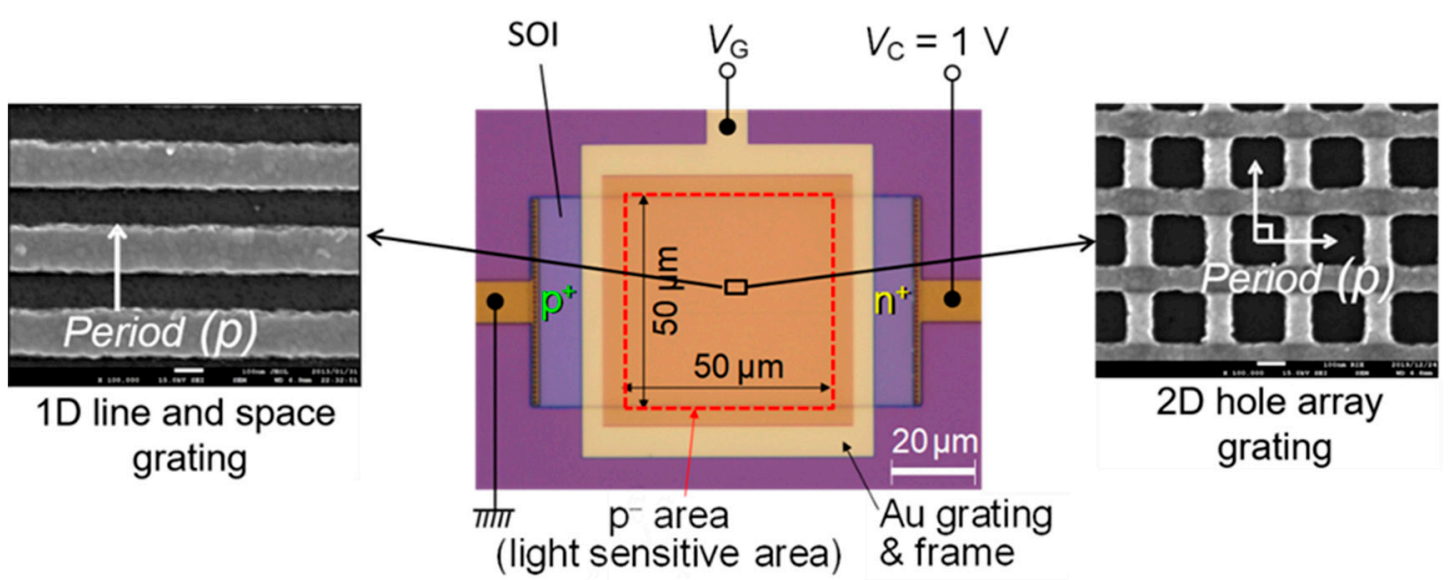

Figure 2. Top view (optical and scanning-electron micrographs) of the fabricated device with the 1D line and space grating and a 2D hole array grating reproduced from [22].

\section{Principle of Angle Detection}

The basic principle of peak angle detection provided by the device is explained in this section. The SOI photodiode functions as a waveguide, as its structure is similar to a symmetrical slab waveguide where the Si layer acts as the core with high refractive index and the gate oxide and the BOX layer act as claddings with a low refractive index $\left(\mathrm{SiO}_{2}\right)$. In Figure 3a, a representation of the waveguide modes in the SOI slab is shown. The main contribution of the SP antenna in the enhancement of light absorption is caused by the strong coupling between the enhanced diffracted light and the propagation mode in the SOI layer. 


\section{SOI slab}

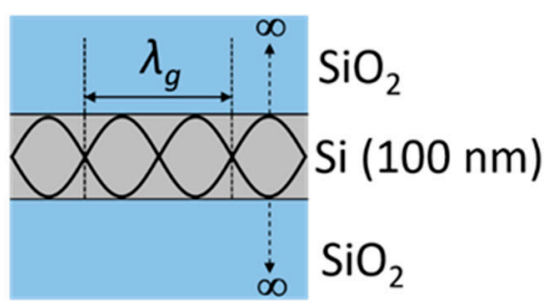

(a)

(b)

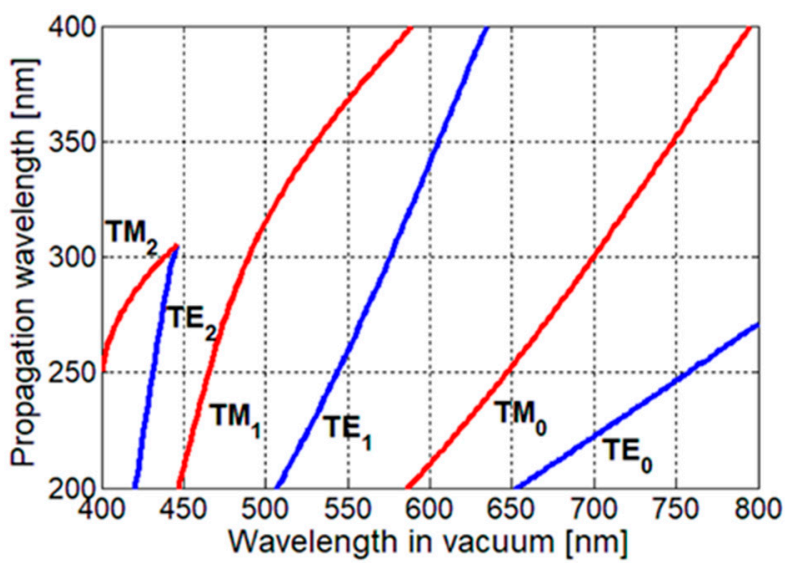

Figure 3. (a) The three-layered symmetrical slab waveguide consists of a 100-nm-thick $\mathrm{Si}$ core and $\mathrm{SiO}_{2}$ claddings; (b) Propagation wavelengths of the waveguide modes in the SOI layer calculated by the dispersion relation vs. the free space wavelength.

The angle-selective enhancement of the device occurs when the phase matching condition between the diffracted mode of the antenna and the propagation mode of the SOI waveguide is satisfied. Through the concept of phase matching condition and the relation between the grating period of the SP antenna, the waveguide mode of SOI PD, the wavelength, and the incident angle $(\phi, \theta)$ of light could be formalized and used to predict the directivity of the SOI PD with the SP antenna towards the incoming light.

To analyze the basic principle in detail, we first considered the dispersion relation for the symmetrical Si-core slab waveguide. The propagation wavelength of the waveguide mode can be predicted by using the dispersion relation based on the following transcendental equations [23]:

For TE modes,

$$
\tan \left(\frac{h t_{\text {core }}}{2}-m \frac{\pi}{2}\right)=\frac{\sqrt{V^{2}-h^{2} t_{\text {core }}^{2}}}{h t_{\text {core }}}(\mathrm{m}=0,1,2, \ldots,)
$$

For TM modes,

$$
\tan \left(\frac{h t_{\text {core }}}{2}-m \frac{\pi}{2}\right)=\frac{n_{\text {core }}^{2}}{n_{\text {clad }}^{2}} \frac{\sqrt{V^{2}-h^{2} t_{\text {core }}^{2}}}{h t_{\text {core }}}(\mathrm{m}=0,1,2, \ldots,)
$$

where,

$$
h=\sqrt{\left(\frac{2 \pi n_{\text {core }}}{\lambda}\right)^{2}-\left(\frac{2 \pi}{\lambda_{g}}\right)^{2}}, \quad V=\frac{2 \pi}{\lambda} t_{\text {core }} \sqrt{n_{\text {core }}^{2}-n_{\text {clad }}^{2}}
$$

$t_{\text {core }}$ is Si core thickness (100 $\mathrm{nm}$ in our design), while $n_{\text {core }}$ and $n_{\text {clad }}$ are the refractive indices for Si core and $\mathrm{SiO}_{2}$ claddings, respectively. Especially for $n_{\text {core, }}$ the wavelength dependence is considered. $\lambda$ and $\lambda_{\mathrm{g}}$ are the free space wavelength and the propagation wavelength, respectively. Figure $3 \mathrm{~b}$ shows the calculated propagation wavelength of the waveguide in the visible light wavelength range.

We then considered a monochromatic incident light where the wavelength of $\lambda$ is tilted in the $x-z$ plane with the angle of $\theta_{x z}$ and the peak angle could be obtained from the previously noted dispersion relation and the following phase matching condition [20]. Figure 4 shows the schematics of the phase matching condition between the diffracted light from SP antenna and the waveguiding mode in the SOI layer when the incident angle is elevated. Due to the periodic arrangement of gold line and space, 
phase difference $\Delta$ occurs between the rays entering the adjacent gold lines in the antenna, when the incident angle is tilted, and $\Delta$ is given by

$$
\Delta=p(2 \pi / \lambda) \sin \theta_{x z} .
$$
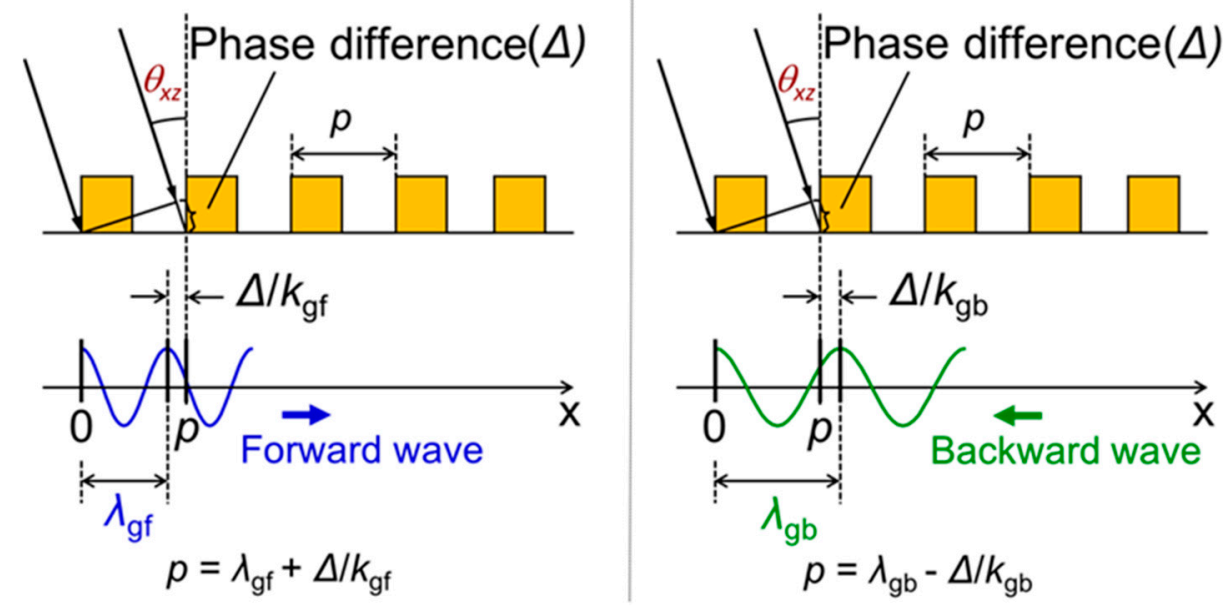

Figure 4. Phase matching conditions between the diffracted wave from antenna surface and the waveguide mode in the SOI layer for forward and backward waves reproduced from [22]. $k_{g f}=2 \pi / \lambda_{g f}$ and $k_{g b}=2 \pi / \lambda_{g b}$ are the wavenumbers for forward and backward waves, respectively. In the case of $y$-direction, $\theta_{y z}$ is considered instead of $\theta_{x z}$.

This phase difference is responsible for propagation of two different waves in the SOI waveguide: forward and backward waves. The forward and backward waves have shorter and longer wavelengths, respectively, with respect to the grating period of the antenna. At a specific angle, the SP antenna strongly couples with the diffracted light from the antenna surface to the propagating wave in the SOI layer when the phase matching condition is satisfied. This phase matching condition occurs at different angles correspondingly with the grating period for forward and backward waves. Based on this physical concept, the mathematical equations were modeled for predicting the peak angle in $x-z$ plane is $\left(\theta_{x z}\right)$ as follows:

For forward waves,

$$
\theta_{x z}=\sin ^{-1} \lambda\left[\left(1 / \lambda_{g f}\right)-(1 / p)\right]
$$

and for backward waves,

$$
\theta_{x z}=\sin ^{-1} \lambda\left[(1 / p)-\left(1 / \lambda_{g b}\right)\right]
$$

where $\lambda_{\mathrm{gf}}$ and $\lambda_{\mathrm{gb}}$ are the propagation wavelengths in forward and backward directions. These theoretical peak angles can be designed using the following procedure. At first, the propagation wavelength $\lambda_{\mathrm{g}}$ for the free space incident wavelength $\lambda$ is obtained from the dispersion relation, as shown in Figure $3 \mathrm{~b}$. Substituting the calculated value $\lambda_{\mathrm{g}}$ for $\lambda_{\mathrm{gf}}$ or $\lambda_{\mathrm{gb}}$ in Equations (4) or (5), the peak angle $\theta_{x z}$ of forward or backward waves can be obtained for each grating period at a fixed incident wavelength.

When the phase matching condition between the waves in the antenna and the SOI layer is considered in the $y$-direction, the same manner can be adopted and $\theta_{y z}$ is considered instead of $\theta_{x z}$.

In the above equations, the elevation angle for a fixed azimuth angle could be predicted. i.e., when $\phi$ $=0$ and $90^{\circ}, \theta_{x z}$ and $\theta_{y z}$ tilting occurs, respectively. For a 2D angular mapping, azimuth $(\phi)$ angle detection also needs to be precisely predicted in addition to the elevation $(\theta)$ angle. This can be calculated by considering Equations (1) and (2).

It is important to consider the design of both types of SP antenna in Figure 1b,c. The structure of $1 \mathrm{D} \mathrm{L} / \mathrm{S}$ grating has a periodic arrangement of line and space along the $x$ direction, whereas along 
the $y$ direction, a straight line is present. This phase matching condition effect appears only along the $x-z$ plane, i.e., when the light direction is perpendicular to the grating orientation, whereas along the $y-z$ plane, angle tilting has no effect on the optical phenomenon of detecting the angle, i.e., when the direction is parallel to the grating orientation. Thus, $1 \mathrm{D}$ grating would exhibit strong polarization sensitive angle detection.

In the case of 2D hole array grating, the periodic arrangement of holes is present in both the directions, $x$ and $y$ and due to its square latticed arrangement, the alignment of holes is also similar in both of the directions. Due to their symmetry, two phase matching conditions in $x-z$ and $y-z$ planes were imposed, as discussed in Section 4.2.

\section{Results and Discussions}

\section{1. $1 D$ L/S SP Antenna}

We first show the light sensitivity in the SOI PD with 1D L/S SP antenna to the elevation angle $(\theta)$ tilting at a fixed azimuth angle $(\phi)$ and polarization angle $\left(\phi_{\text {pol }}\right)$. To predict the performance of our devices quantitatively, the absorption efficiency in the SOI layer was calculated by using the FDTD method. In this simulation, we used the Lorentz-Drude oscillator model and the single-pole Lorentz model to introduce the dielectric constants depending on wavelength of metals (Au and Ti) and silicon, respectively. These parameters are the same as the ones in [18]. The relative permittivity of $\mathrm{SiO}_{2}$ for the layers of BOX and gate oxide were fixed at 2.13. In order to reduce the computation costs, the periodic boundary condition and the absorbing boundary condition were adopted. Especially for absorbing the boundary condition, we chose the perfectly matching layer (PML). FDTD simulations mainly evaluate the absorption efficiency based on the ratio of the power absorbed in the SOI layer to the one of incident light, because the absorption efficiency in the SOI layer corresponds to the external $\mathrm{QE}$ of PD when it is assumed that the internal QE of PD is unity. The absorption power in the SOI layer can be obtained by calculating the term of conduction loss in the Maxwell equation or by subtracting the power passing through at the lower interface of SOI from the upper interface [17]. Figure 5a shows the FDTD results of absorption efficiency in the SOI layer as a function of elevation angle $\theta$ for various grating periods $p$ ranging from 285 to $340 \mathrm{~nm}$ with an interval of $5 \mathrm{~nm}$. The incidence was a TM-polarized monochromatic light with the wavelength of $685 \mathrm{~nm}$, and the incident direction was moved along the perpendicular one to the $\mathrm{L} / \mathrm{S}$ orientation, i.e., at $\phi=\phi_{\mathrm{pol}}=90^{\circ}$. An elevation angle dependence of absorption efficiency with a sharp peak was observed at each period, and the peak elevation angle clearly shifted due to the change of the grating period. The peak heights except for $p=$ $285 \mathrm{~nm}$ were almost the same, and the peak height in the case of $p=285 \mathrm{~nm}$ was about 0.1 larger than the others. The propagation wavelength of the symmetrical waveguide with 100-nm-thick Si for the incident wavelength of $685 \mathrm{~nm}$ was $285.5 \mathrm{~nm}$ calculated from Equation (2). When the propagation wavelength and the grating period $p$ of SP antenna is matched, strong coupling of the diffracted light with the SOI waveguide mode occurs for normal incidence. This characteristic has been already discussed in detail in our previous research [17-20]. In Figure 5b, the peak elevation angle for the different grating period $p$ under the TM waveguide mode was predicted by the FDTD calculation and phase matching condition for the forward waves in order to check their correlation. Similar trends were obtained so that peak elevation angle linearly increases as the grating period $p$ increases and the peak positions coincide exactly with each other. The output response of each pixel could be modeled by using a simple Gaussian distribution equation. Thus, it can be shown that $p$ can tune the peak elevation angle. The peak angle could also be controlled by varying the thickness of the SOI layer. However, we fixed the $t_{\mathrm{SOI}}$ to facilitate monolithic integration of PD with different grating periods for achieving angle detection with different characteristics. Note that the phase matching condition can estimate a peak elevation angle only, but FDTD results can investigate more details, including absorption efficiency, peak width, and so on. Considering the actual incident angle detection, we had to discuss not only the elevation angle but also the azimuth angle. In addition, the light in nature 
consists of polarized rays. Thus, the elevation-azimuth angle dependence of QE for different types of polarization was investigated for a rich collection of light information for image processing applications. A distribution of absorption efficiency based on polar coordinate system was used as a spatial pattern. This spatial pattern is convenient to represent the complete spherical optical information emanating from a point in space. Figure $5 \mathrm{c}$ shows the FDTD results of the spatial pattern at $p=300 \mathrm{~nm}$ and $\phi_{\text {pol }}=90^{\circ}$. Figure $5 \mathrm{~d}$ shows the peak position of azimuth and elevation angles based on the phase matching condition. The spatial pattern based on the phase matching condition has good agreement with the one estimated by FDTD calculation, with a small angle deviation of $\sim 0.5^{\circ}$.

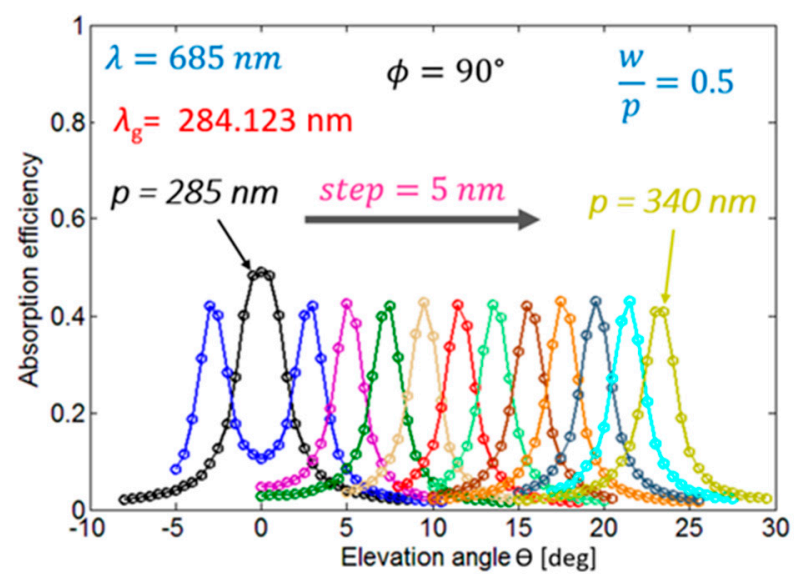

(a)

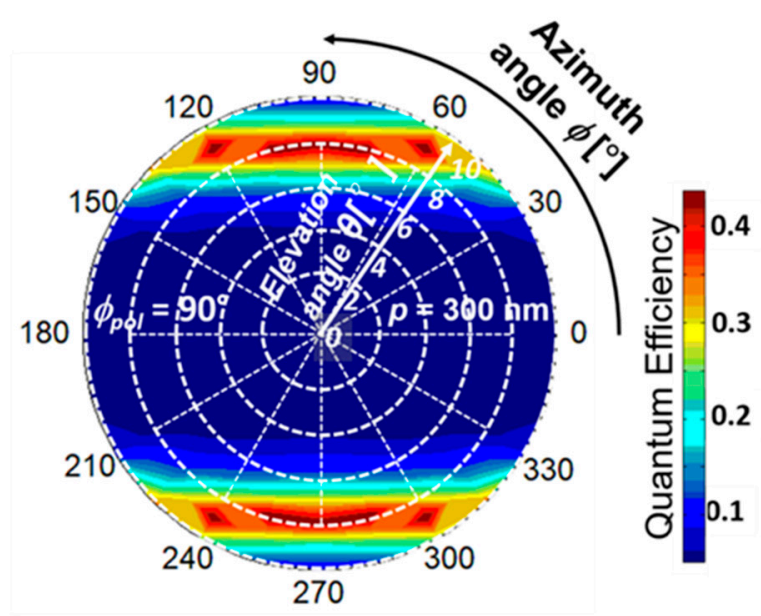

(c)

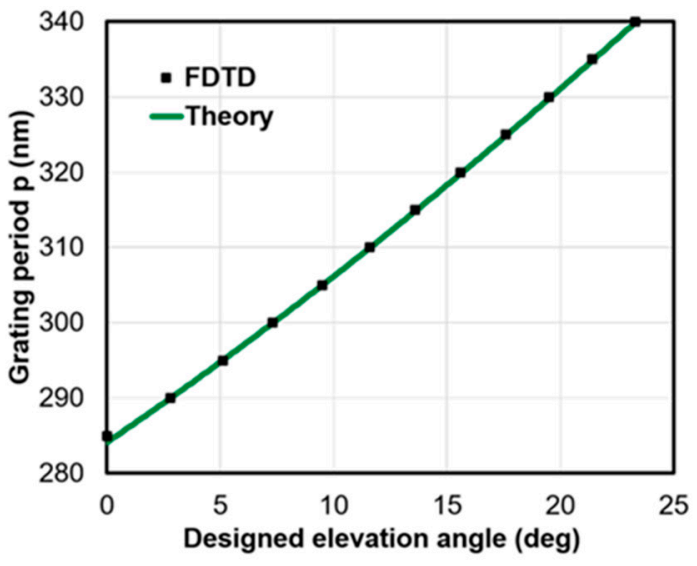

(b)

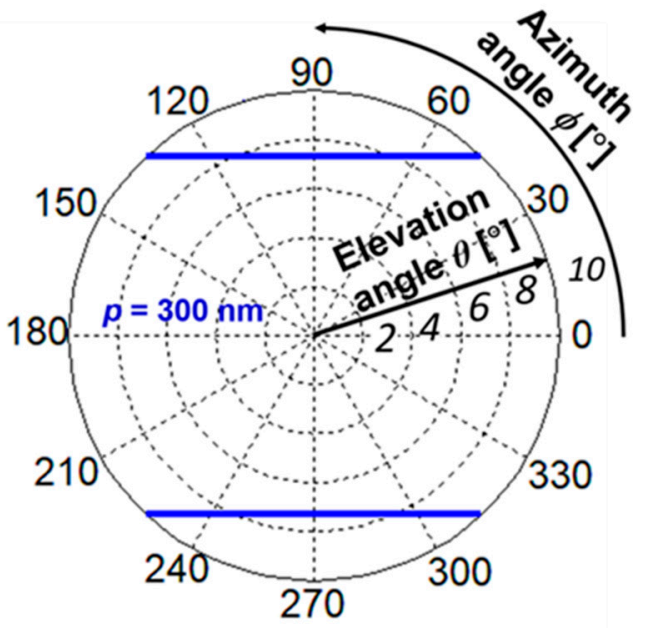

(d)

Figure 5. (a) Absorption efficiency as a function of the elevation angle in SOI PD with $1 D$ L/S SP antenna (FDTD calculation) with various grating periods $p$; (b) Comparison of appropriate grating periods for designed peak elevation angle estimated by FDTD method and phase matching condition. (c) Calculated spatial pattern of absorption efficiency at $\phi_{\text {pol }}=90^{\circ}$ and (d) theoretical spatial pattern of peak incident angles based on the phase matching condition. Grating period is fixed at $p=300 \mathrm{~nm}$.

The SOI PD with 1D L/S antenna is fabricated for measurements, where the thicknesses of SOI, gate oxide, $\mathrm{BOX}, \mathrm{Au}$, and $\mathrm{Ti}$ are $t_{\mathrm{SOI}}=105 \mathrm{~nm}, t_{\mathrm{ox}}=100 \mathrm{~nm}, t_{\mathrm{BOX}}=400 \mathrm{~nm}, t_{\mathrm{Au}}=50 \mathrm{~nm}$, and $t_{\mathrm{Ti}}=5 \mathrm{~nm}$, respectively. The grating period and width are $p=286 \mathrm{~nm}$ and $w=143 \mathrm{~nm}$, respectively. The spatial pattern was measured for the elevation angles from 0 to $8^{\circ}$ and the azimuth angles from 0 to $360^{\circ}$ under three different polarizations: $\phi_{\text {pol }}=0,45^{\circ}$, and $90^{\circ}$. Figure 6 shows our measurement system. The light source is the solid-state laser, which emits a linearly-polarized light with a wavelength of $685 \mathrm{~nm}$. 
The half-wave plate was used to calibrate a basic axis for the incident polarization angle $\phi_{\text {pol }}$ from the grating orientation of PDs, and $\phi_{\mathrm{pol}}$ was adjusted by the rotation stage. The collimated light by beam expander was irradiated to the PDs. The azimuth angle $\phi$ and the elevation angle $\theta$ were adjusted by moving the $\alpha \beta$ goniometer stage. The electrical characteristics of fabricated PD were measured by using a semiconductor parameter analyzer. Here, the photocurrent is evaluated when the reverse bias voltage of $-1 \mathrm{~V}$ is applied between the anode $\left(\mathrm{p}^{+}\right.$region) and the cathode $\left(\mathrm{n}^{+}\right.$region) electrodes. Since our metallic SP antenna can be used for a gate electrode, the gate bias voltage was applied to control the carrier distribution. Combined with the substrate bias voltage, the depletion region can be expanded entirely in a $\mathrm{p}^{-}$light sensitive region [18]. In this paper, the gate voltage at $V_{\mathrm{G}}=7 \mathrm{~V}$ and the substrate voltage at $V_{\text {SUB }}=-25 \mathrm{~V}$ are commonly used, including in the next subsection. The measured spatial patterns for different polarization angles (i) $\phi_{\text {pol }}=0$, (ii) $45^{\circ}$ and (iii) $90^{\circ}$ are shown in Figure $7 \mathrm{a}$. The contour map with two-fold symmetry appeared in all the cases. When the incident light with the electric field perpendicular to the grating orientation (TM polarization) is illuminated, strong coupling occurs with the TM fundamental waveguide mode $\left(\mathrm{TM}_{0}\right)$ in the SOI layer [21-24]. The QE reaches a maximum of 0.4 as predicted in the FDTD results when $\phi_{\mathrm{pol}}=90^{\circ}$, and reaches 0.2 (half value in the case of $\phi_{\text {pol }}=90^{\circ}$ ) when $\phi_{\text {pol }}=45^{\circ}$. Thus, the QE is higher in the case of perpendicular polarization and gradually decreases as the polarization decreases to zero. Such polarization dependence has been already described in our previous work, and the maximum QE for each polarization angle in a spatial pattern, denoted by $\eta_{\max }\left(\phi_{\text {pol }}\right)$, can be roughly estimated by $\eta_{\max }\left(\phi_{\text {pol }}\right)=\eta_{\max }\left(\phi_{\text {pol }}=90^{\circ}\right)$ $\cos ^{2} \phi_{\text {pol }}$ [22]. Figure $7 \mathrm{~b}$ shows the theoretical spatial pattern for the grating period $p=286 \mathrm{~nm}$ based on the phase matching condition. It is shown that the two-fold symmetry was also obtained that was similar to the experimental demonstration. For the case of $\phi_{\text {pol }}=0$, (TE polarization), the coupling occurs with the TE waveguide modes propagating in the SOI slab. However, the peaks corresponding to this polarization are invisible in the elevation angle range of these measurements.

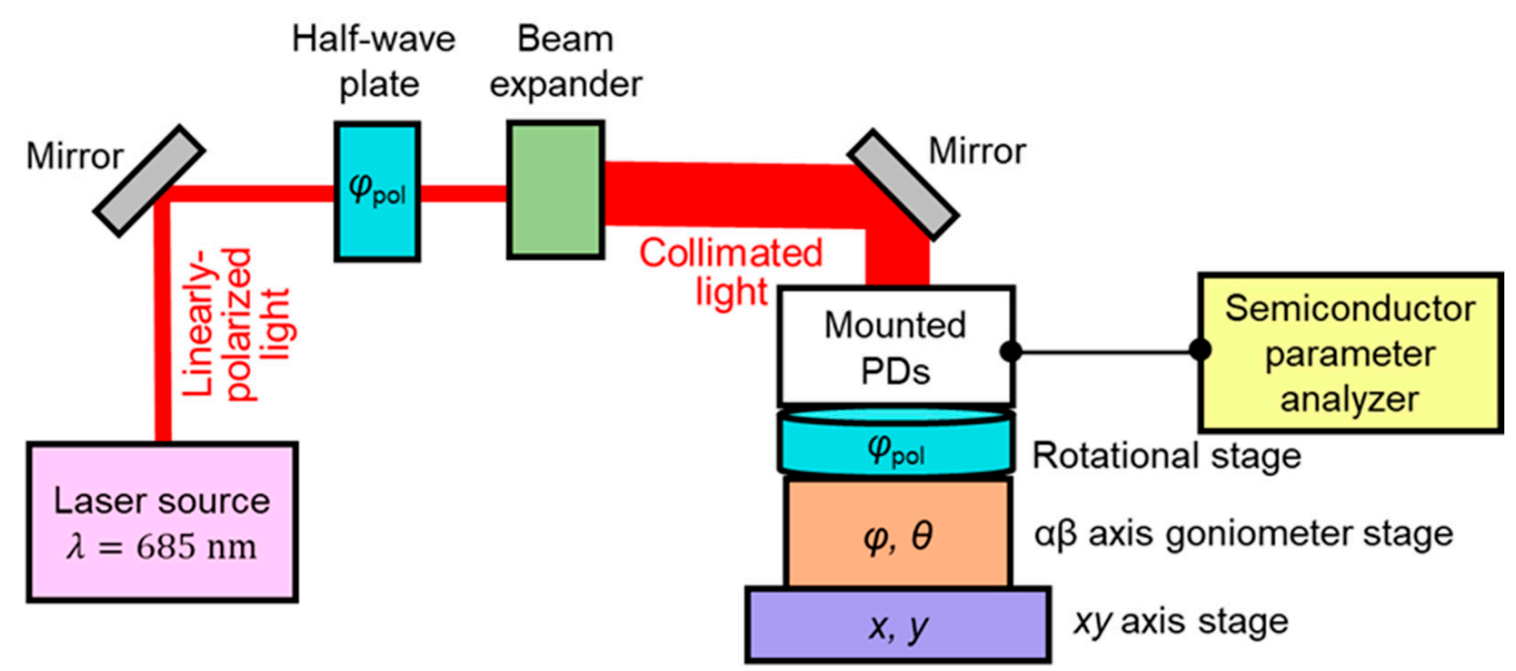

Figure 6. Measurement system for directivity of external QE in SOI PD with a SP antenna. 


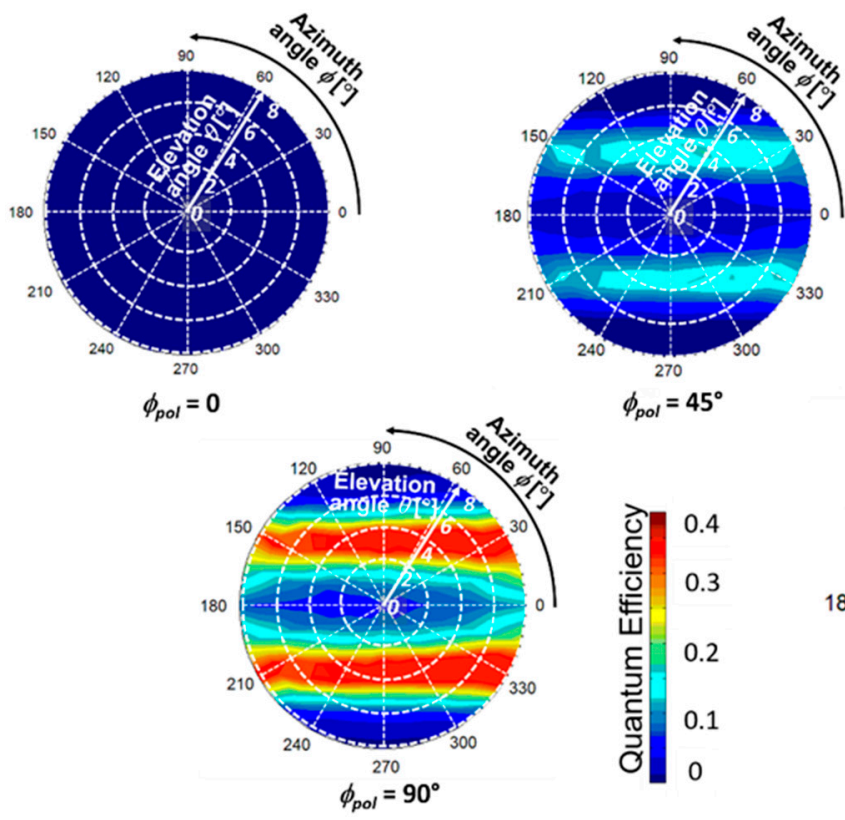

(a)

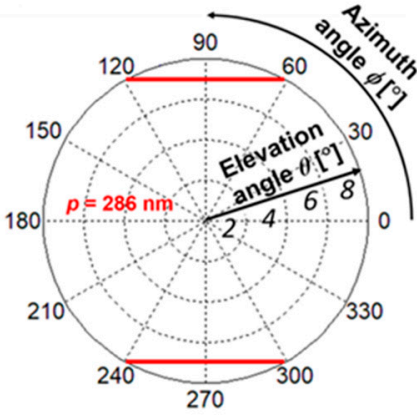

(b)

Figure 7. (a) Measured spatial patterns in SOI PD with a 1D L/S SP antenna for different polarizations (i) $\phi_{\text {pol }}=0,45^{\circ}$ and $90^{\circ}$; (b) theoretical spatial pattern of peak incident angle based on phase matching condition to verify the measurements reproduced from [22]. The grating period was fixed at $p=286 \mathrm{~nm}$.

\subsection{D Hole Array SP Antenna}

Here, the type of SP antenna was changed to a 2D hole array, and its spatial pattern depending on incident polarization is discussed. As discussed in the previous subsection, the FDTD calculation was performed before obtaining measurements for the PD with a grating period of $p=300 \mathrm{~nm}$ and SOI thickness of $t_{S O I}=100 \mathrm{~nm}$. Figure 8 a shows the spatial pattern for different polarization of $\phi_{\text {pol }}=0$, $45^{\circ}$, and $90^{\circ}$. In the cases of $\phi_{\text {pol }}=0$ and $90^{\circ}$, the FDTD spatial patterns have two-fold symmetry in the vertical and the horizontal directions, respectively. However, in the case of $\phi_{\text {pol }}=45^{\circ}$, the spatial pattern with four-fold symmetry appears. This pattern may be caused by the superposition of the patterns of $\phi_{\mathrm{pol}}=0$ and $90^{\circ}$. The peak elevation angle trend in $\phi_{\mathrm{pol}}=90^{\circ}$ is the inverse of that of the $\phi_{\text {pol }}=0$ as the azimuth angle increases. Thus, peak angle sensitivity is highly symmetric with the parallel, and perpendicular calculation is also further expanded to predict the 2D angular distribution and plotted in polar coordinates with the spatial pattern representation, as shown in Figure $8 \mathrm{~b}$. The elevation-azimuth angular information mapping by the theory and numerical predictions were in good agreement, ensuring that the peak angle appears based on the optical concept and phase matching condition.

For the demonstration of polarization dependence in SOI PD with 2D hole array to elevation-azimuth angle detectivity, the external quantum efficiency was measured for the polarizations, $\phi_{\text {pol }}=0,45$, and $90^{\circ}$ and the corresponding spatial patterns are represented in Figure 9 a. The polarization 0 and $90^{\circ}$ shows a two-fold symmetry and follows the inverse trend of peak sensitivity with each other. Due to the high symmetric structure of the 2D SP antenna, strong coupling occurs for both the parallel and perpendicular polarizations. Also, the polarization $45^{\circ}$ exhibits a superposition of the polarizations 0 and $90^{\circ}$. The experimental spatial pattern of SOI PD with 2D hole array SP antenna trend is similar to that observed in the numerical prediction. The theoretical spatial pattern for SOI PD with 2D SP antenna with $p=286 \mathrm{~nm}$ and $t_{S O I}=105 \mathrm{~nm}$ for the TM mode is shown in Figure $9 \mathrm{~b}$. The peak angles of the theory and the experiment are close enough with a small variation, which is explained in Figure 10. The spatial patterns for both the devices clearly show the capability to detect both azimuth and elevation angles and polarization of the incoming light. Each device is capable of 
decoding an angular information with a high angular resolution of $\sim 2^{\circ}$. The spatial pattern shows the full sphere of optical information impinging on a point in space and reveals the angle detection in both elevation and azimuth directions. LS grating owing to its $1 \mathrm{D}$ periodicity with high QE (45\%) exhibits a strong polarization sensitive behavior and stands as a promising candidate for developing pixels for polarization vision applications. Hole array grating has the advantage of detecting angles with polarization independent behavior.

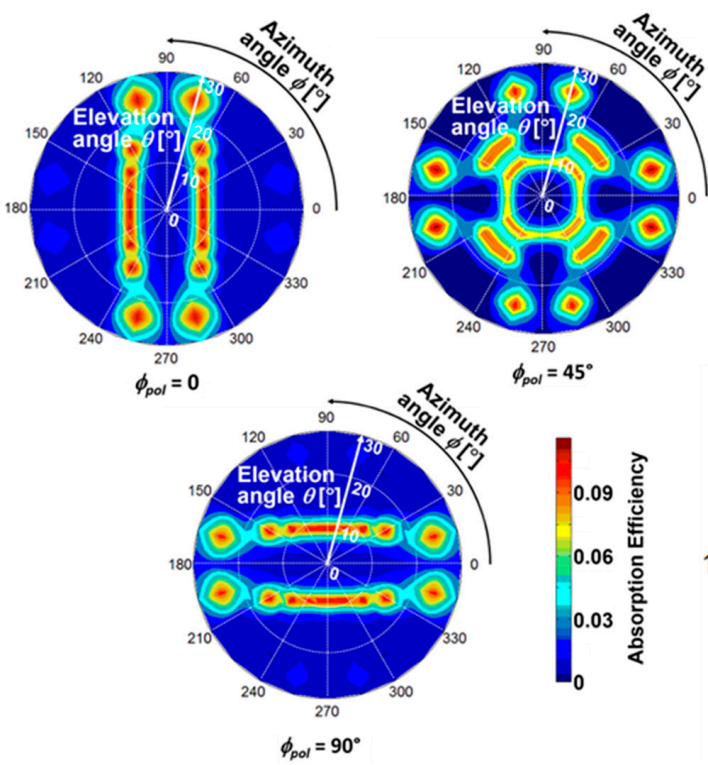

(a)

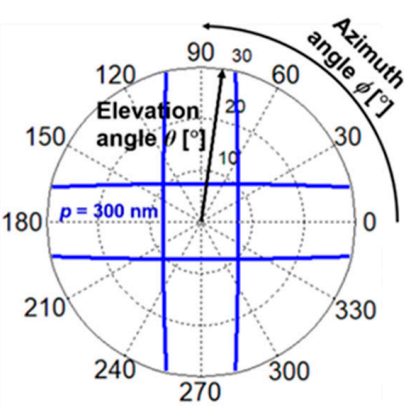

(b)

Figure 8. Numerical prediction of SOI PD with a 2D SP antenna. Polarization dependence of 2D angular detection in SOI PD with a 2D hole array grating with the period $p=300 \mathrm{~nm}$. (a) FDTD spatial pattern showing $2 \mathrm{D}$ angular mapping for different polarizations, $\phi_{\mathrm{pol}}=0,45^{\circ}$, and $90^{\circ}$; (b) theoretical spatial pattern for SOI PD for a 2D hole array with a grating period of $p=300 \mathrm{~nm}$ reproduced from [22].
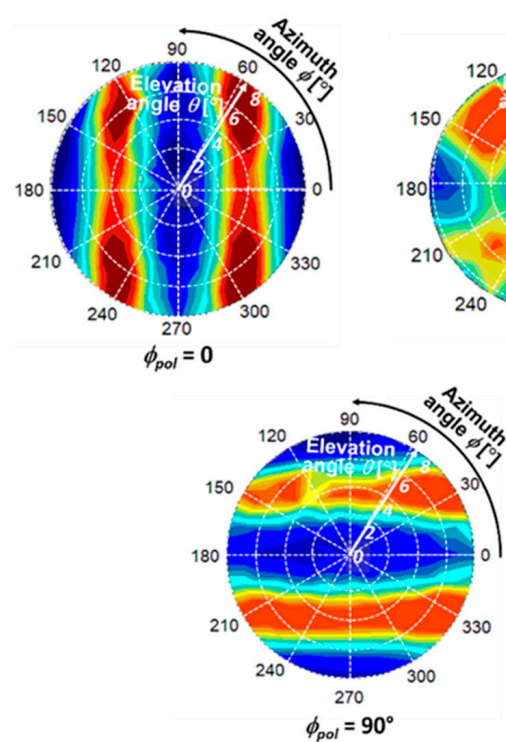

(a)
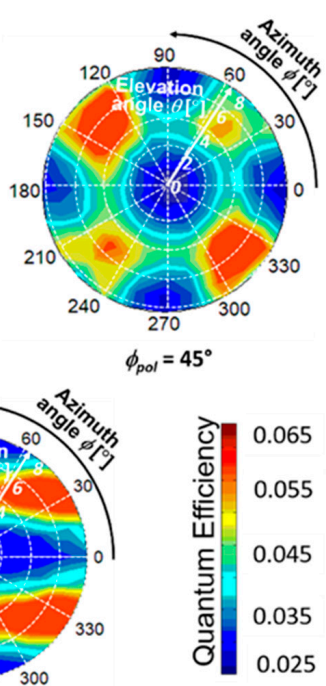

0.025

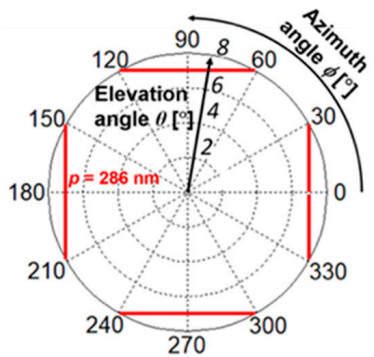

(b)

Figure 9. (a) Measured spatial patterns in SOI PD with a 2D hole array SP antenna for different polarizations of (i) $\phi_{\text {pol }}=0,45^{\circ}$, and $90^{\circ}$; (b) theoretical spatial pattern of a peak incident angle based on phase matching conditions to verify the measurements reproduced from [22]. The grating period is fixed at $p=286 \mathrm{~nm}$. 


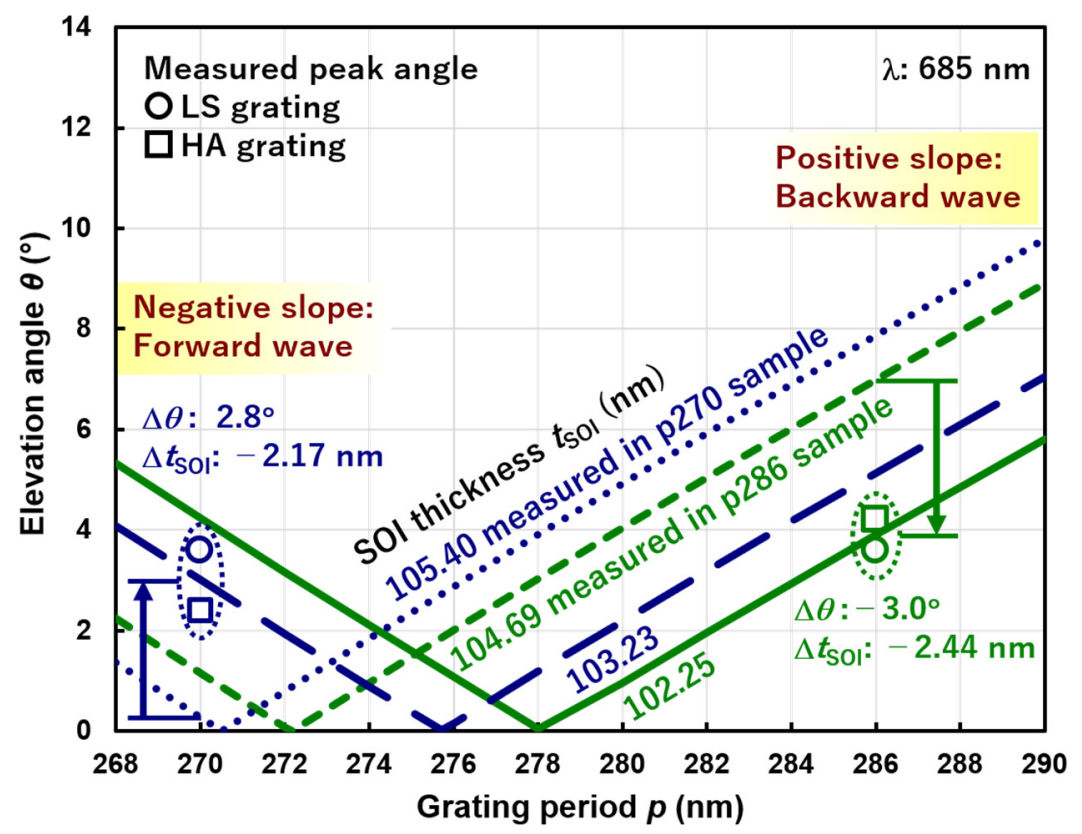

Figure 10. Comparison between measured peak elevation angles (circles and squares indicate LS and HA grating, respectively) and theoretical lines based on the phase matching condition. Samples with the grating periods $p=270$ (blue) and 286 (green) nm have different measured SOI thicknesses $t_{\mathrm{SOI}}=105.40$ and $104.69 \mathrm{~nm}$, (dotted lines) respectively. Theoretical lines for measured and estimated (solid lines) SOI thicknesses for $p=270$ and $286 \mathrm{~nm}$ are shown. The arrows indicate the shift of measured angles from the estimated ones.

Figure 10 compares the measured peak elevation angles (indicated by symbols as shown in the legend) for two grating periods of $p=270$ (blue) and 286 (green) nm, and grating types of 1D L/S (circle) and 2D hole array (square) with the theoretical lines based on the phase matching condition for measured (dotted lines) and estimated (solid lines) $t_{\text {SOI }}$. The SOI thicknesses were measured by the spectroscopic reflectometer (Otsuka Electronics Co., Ltd., Tokyo, Japan, FE-3000) just before the fabrication of the SP antenna. The measured peak angles are $2.8^{\circ}$ larger and $3.0^{\circ}$ smaller than the theoretical ones for $p=270$ and $286 \mathrm{~nm}$, respectively. These deviations from the theory can be explained by the reduction of $t_{\mathrm{SOI}}$ by 2.17 and $2.44 \mathrm{~nm}$, respectively. Note that the decrease in $t_{\mathrm{SOI}}$ results in the increase and the decrease of the peak elevation angles for forward and backward waves, respectively. In order to understand the discrepancy in $t_{\mathrm{SOI}}$, optical constants of $\mathrm{Si}$ and $\mathrm{SiO}_{2}$ in the wavelength range of $230-800 \mathrm{~nm}$ used in the $t_{\mathrm{SO}}$ measurement need to be analyzed as a future task. These results indicate that the accurate control of $t_{\mathrm{SO}}$ is necessary to reproduce the peak incident angles, which is not so difficult for advanced SOI CMOS technology. Also note that the material, i.e., optical constants, width, and thickness of the SP antenna do not affect the peak angles [19], which is beneficial for attaining reproducibility.

\section{Conclusions}

The effect of polarization on detectivity of light sensitivity in our proposed SOI PD with two types of SP antenna composed of 1D L/S grating and 2D hole array grating was investigated by using theoretical and simulated predictions, and experimental demonstration. While the conventional ASP focuses only on the local angular distribution of light, our proposed devices are capable of exhibiting elevation-azimuth angular detection. In addition, the spatial pattern representation was very unique for understanding the dependences of not only the elevation-azimuth angle but also polarization. The device with $1 \mathrm{D}$ grating shows angle detection with strong polarization selectivity. The 2D grating which was designed by simply changing the layout of the antenna by overlapping the 1D grating in two orthogonal directions, which exhibits angle detection with polarization insensitive behavior. The SP 
antenna played an effective role in affecting both the incident angle and polarization of light caused by the coupling between the diffracted light from the SP antenna and the waveguide mode in the SOI layer when the phase matching condition was satisfied. Several pixels with different grating periods could be integrated in a single chip for tuning multiple incident angles for sufficiently extracting the angle features of the image, and contributed to the development of an advanced ASP as a key component of plenoptic cameras that enable interesting applications such as three-dimensional (3D) imaging, depth-of-field extension, lensless imaging, and so on. 1D grating especially opens an opportunity for developing tiny pixels to enable applications based on polarization vision due to its polarization sensitive behavior.

Author Contributions: H.I. and H.S. conceived of the presented idea and developed the theoretical concept. A.N. and H.S. have contributed to the computation. A.N. have contributed to the data analysis and drafting the manuscript, which was further revised by H.S. and H.I. S.H. and H.S. designed, fabricated, and measured the devices. H.I. and A.P.P. cooperatively supervised the project. All authors have read and agreed to the published version of the manuscript.

Funding: This research was funded by JSPS KAKENHI (Grant No. 18K04261), the Cooperative Research Project of the Research Center for Biomedical Engineering with RIE, Shizuoka University, and the Cooperative Research Project Program of RIEC, Tohoku University.

Acknowledgments: The authors are indebted to the Solid State Division of Hamamatsu Photonics K.K. for providing the SOI photodiodes before fabrication of the SP antenna.

Conflicts of Interest: The authors declare no conflict of interest.

\section{References}

1. Sivaramakrishnan, S.; Wang, A.; Gill, P.; Molnar, A. Design and characterization of enhanced angle sensitive pixels. IEEE Trans. Electron Devices 2016, 63, 113-119. [CrossRef]

2. Papageorgiou, E.P.; Boser, B.E.; Anwar, M. An angle-selective CMOS imager with on-chip micro-collimators for blur reduction in near-field cell imaging. In Proceedings of the 2016 IEEE 29th International Conference on Micro Electro Mechanical Systems (MEMS), Shangai, China, 24-28 January 2016; pp. 337-340.

3. Wang, P.; Menon, R. Computational snapshot angular-spectral lensless imaging. arXiv 2017, arXiv:1707.08104.

4. Yi, S.; Zhou, M.; Yu, Z.; Fan, P.; Behdad, N.; Lin, D.; Wang, K.X.; Fan, S.; Brongersma, M. Subwavelength angle-sensing photodetectors inspired by directional hearing in small animals. Nat. Nanotechnol. 2018, 13, 1143-1147. [CrossRef] [PubMed]

5. Adelson, E.H.; Bergen, J.R. The plenoptic function and the elements of early vision. Comput. Models Vis. Process. 1991, 3-20.

6. Pereira, F.; Da Silva, E.A.B. Efficient plenoptic imaging representation: Why do we need it? In Proceedings of the 2016 IEEE International Conference on Multimedia and Expo (ICME), Seattle, WA, USA, 11-15 July 2016; pp. 1-6.

7. Gershun, A. The Light Field. J. Math. Phys. 1939, 18, 51-151. [CrossRef]

8. Fiss, J.; Curless, B.; Szeliski, R. Refocusing plenoptic images using depth-adaptive splatting. In Proceedings of the 2014 IEEE international conference on computational photography (ICCP), Santa Clara, CA, USA, 2-4 May 2014; pp. 1-9.

9. Shroff, S.A.; Berkner, K. Image formation analysis and high resolution image reconstruction for plenoptic imaging systems. Appl. Opt. 2013, 52, D22-D31. [CrossRef] [PubMed]

10. Wetzstein, G.; Ihrke, I.; Lanman, D.; Heidrich, W. Computational plenoptic imaging. Comput. Graph. Forum 2011, 30, 2397-2426. [CrossRef]

11. Levoy, M. Light fields and computational imaging. Computer 2006, 39, 46-55. [CrossRef]

12. McMillan, L.; Bishop, G. Plenoptic modeling: An image-based rendering system. In Proceedings of the 22nd annual conference on Computer graphics and interactive techniques, Los Angeles, California, USA, 6-11 August 1995; pp. 39-46.

13. Radwell, N.; Selyem, A.; Mertens, L.; Edgar, M.P.; Padgett, M.J. Hybrid 3D ranging and velocity tracking system combining multi-view cameras and simple LiDAR. Sci. Rep. 2019, 9, 1-7. [CrossRef] [PubMed]

14. Kuo, G.; Antipa, N.; Ng, R.; Waller, L. DiffuserCam: Diffuser-based lensless cameras. In Proceedings of the Computational Optical Sensing and Imaging, San Francisco, California, USA, 26-29 June 2017; p. CTu3B-2. 
15. Stork, D.G.; Gill, P.R. Lensless Ultra-Miniature CMOS Computational imagers and sensors. In Proceedings of the Seventh International Conference on Sensor Technologies and Applications (SENSORCOMM 2013), Barcelona, Spain, 25-31 August 2013; pp. 186-190.

16. Wang, A.; Molnar, A. A light-field image sensor in 180 nm CMOS. IEEE J. Solid-State Circuits 2012, 47, $257-271$. [CrossRef]

17. Satoh, H.; Inokawa, H. Surface plasmon antenna with gold line and space grating for enhanced visible light detection by a silicon-on-insulator metal-oxide-semiconductor photodiode. IEEE Trans. Nanotechnol. 2012, 11, 346-351. [CrossRef]

18. Satoh, H.; Ono, A.; Inokawa, H. Enhanced visible light sensitivity by gold line-and-space grating gate electrode in thin silicon-on-insulator p-n junction photodiode. IEEE Trans. Electron Devices. 2013, 60, 812-818. [CrossRef]

19. Satoh, H.; Kawakubo, K.; Ono, A.; Inokawa, H. Material Dependence of Metal Grating on SOI Photodiode for Enhanced Quantum Efficiency. IEEE Photon. Technol. Lett. 2013, 25, 1133-1135. [CrossRef]

20. Inokawa, H.; Satoh, H.; Kawakubo, K.; Ono, A. Enhancement of SOI photodiode sensitivity by aluminium grating. ECS Transactions 2013, 53, 127. [CrossRef]

21. Nagarajan, A.; Hara, S.; Satoh, H.; Panchanathan, A.P.; Inokawa, H. Directivity of SOI Photodiode with Gold Surface Plasmon Antenna. In Proceedings of the 2019 Silicon Nanoelectronics Workshop (SNW), Kyoto, Japan, 9-10 June 2019; pp. 121-122.

22. Nagarajan, A.; Hara, S.; Satoh, H.; Panchanathan, A.P.; Inokawa, H. Polarization Dependence of Incident Angle Sensitivity in Soi Photodiode with 2d Hole Array Grating. In Proceedings of the 2020 Silicon Nanoelectronics Workshop (SNW), Honolulu, HI, USA, 13-14 June 2020; pp. 63-64.

23. Nagarajan, A.; Hara, S.; Satoh, H.; Panchanathan, A.P.; Inokawa, H. Angular Selectivity of SOI Photodiode with Surface Plasmon Antenna. IEICE Electron. Expr. 2020, 17, 20200187. [CrossRef]

24. Liu, J.M. Photonic Devices; Cambridge University Press: Cambridge, UK, 2005; pp. 95-99.

(C) 2020 by the authors. Licensee MDPI, Basel, Switzerland. This article is an open access article distributed under the terms and conditions of the Creative Commons Attribution (CC BY) license (http://creativecommons.org/licenses/by/4.0/). 